\title{
Benefícios da fisioterapia no pós-operatório de câncer de mama: estudo de caso
}

\author{
Benefits of physical therapy after surgery \\ for breast cancer: a case study
}

FisiSenectus. Unochapecó Ano 1 - Edição especial - 2013 p. $76-84$

\section{Joyce Wilhelm}

Acadêmica do $9^{\circ}$ semestre do curso de Fisioterapia da Universidade Regional e Integrada do Alto Uruguai e das Missões (URI) - campus de Erechim, joycewilhelm@hotmail.com

\section{Manoele Molossi Bernardi}

Acadêmica do $9^{\circ}$ semestre do curso de Fisioterapia da Universidade Regional e Integrada do Alto Uruguai e das Missões (URI) - campus de Erechim, manoelem.bernardi@yahoo.com.br

\section{Zequiela Russi}

Fisioterapeuta, especialista em Fisioterapia em Oncologia, preceptora do Estágio de Fisioterapia em Oncologia da Universidade Regional Integrada do Alto Uruguai e das Missões (URI) - campus de Erechim, zequirussi@yahoo.com.br

\section{Vanessa Sebben}

Fisioterapeuta, mestre em Envelhecimento Humano, supervisora do Estágio de Fisioterapia em Oncologia da Universidade Regional Integrada do Alto Uruguai e das Missões (URI) campus de Erechim, vanesebben@ibest.com.br

\section{Resumo}

Introdução: O câncer de mama é o tipo de câncer mais comum entre as mulheres, porém se diagnosticado e tratado oportunamente apresenta um prognóstico relativamente bom. Mesmo com as significativas mudanças nas técnicas de tratamento ainda são muitas as consequências físicas e emocionais encontradas nestes pacientes. Por isso, a abordagem multidisciplinar é fundamental, e a fisioterapia representa um importante papel nestes casos preservando e restaurando a integridade cinético-funcional dos órgãos e sistemas. Objetivos: Verificar os benefícios proporcionados por um programa de fisioterapia em uma paciente no pós-operatório de câncer de mama, buscando uma melhora do quadro álgico, da amplitude de movimento e da força muscular. Materiais e métodos: 0 presente estudo foi delineado pelo método qualiquantitativo em saúde, do tipo estudo de caso, realizado na Clínica Escola de Fisioterapia da URI - campus de Erechim, com uma paciente de 60 anos que se encontrava no segundo mês de pós-operatório de câncer de mama direita. Resultados: Ao primeiro encontro a paciente relatou dores em região axilar e de membro superior direito e cervical, além disso, verificou-se diminuição da amplitude de movimento de membros superiores e diminuição de força muscular do membro superior homolateral à cirurgia. o protocolo fisioterapêutico realizado foi baseado nas limitações funcionais avaliadas, e teve duração de 12 sessões. Conclusão: Após esse período se verificou que o protocolo de tratamento fisioterapêutico foi eficaz no pós-operatório de câncer de mama, proporcionando 
diminuição da dor, prevenção do linfedema, aumento da amplitude de movimento de membros superiores, sem alteração na força muscular.

\title{
Palavras-chave
}

Câncer de mama. Fisioterapia. Pós-operatório.

\begin{abstract}
Introduction: Breast cancer is the most common type of cancer among women, but if diagnosed and treated timely presents a relatively good prognosis. Even with the significant changes in treatment techniques are still many physical and emotional consequences found in these patients. Therefore, a multidisciplinary approach is essential, and physiotherapy plays an important role in these cases preserving and restoring the integrity of the kinetic-functional organs and systems. In view of this, the aim of this study was to assess the benefits provided by a program of physical therapy in a patient after surgery for breast cancer, seeking an improvement of pain, range of motion and muscle strength. Materials and Methods: The present study was designed by the method qualiquantitativo health, the case study, conducted at the School of Physiotherapy of the URI - Campus of Erechim, with a 60 year old patient who was in the second month of postoperative cancer right breast. Results: At the first meeting the patient reported pain in the axillary region and right upper limb and neck, in addition, there was decreased range of motion of upper and decreased muscle strength of the upper limb ipsilateral to surgery. The physical therapy protocol was performed based on functional limitations assessed, and lasted 12 sessions. Conclusion: After this period it was found that the physiotherapy treatment protocol was effective in postoperative breast cancer, thereby resulting in less pain, lymphedema prevention, increased range of motion of the upper limbs, no change in muscle strength.
\end{abstract}

\section{Keywords}

Breast cancer. Physiotherapy. Postoperatively.

\section{Introdução}

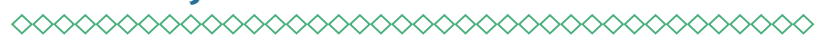

0 câncer de mama é o tipo de câncer mais comum entre as mulheres, sendo o segundo tipo mais frequente no mundo. Apresenta um prognóstico relativamente bom, se diagnosticado e tratado oportunamente. A sobrevida média, na população mundial, após cinco anos é de $61 \%$; entretanto, no Brasil, as taxas de mortalidade continuam elevadas, muito provavelmente porque a doença ainda é diagnosticada em estádios avançados. ${ }^{1}$

Nos últimos 20 anos, as técnicas de tratamento do câncer de mama sofreram significativas mudanças, as cirurgias são menos radicais e a terapêutica complementar (radioterapia, quimioterapia e hormonioterapia) procura estabelecer uma relação adequada entre dosagem, efeitos secundários e eficácia do tratamento.

Entretanto, ainda assim, muitas são as consequências físicas e emocionais desfavoráveis que ocorrem no pós-operatório, como dor, diminuição da amplitude do movimento de membros superiores, fraqueza no membro superior do lado operado, linfedema, complicações cicatriciais, alterações posturais, problemas respiratórios, dentre outros problemas. ${ }^{2}$

Por isso, a abordagem multidisciplinar é fundamental para estes pacientes, tendo em vista uma recuperação global, nos âmbitos físico, psicológico, social e profissional. ${ }^{3}$ Sendo assim, a fisioterapia representa um importante papel nestes casos, já que esta visa preservar e restaurar a integridade cinético-funcional de órgãos e sistemas, assim como prevenir os distúrbios causados pelo tratamento oncológico. ${ }^{4-5}$

Em vista disso, o objetivo deste estudo foi verificar os benefícios proporcionados por um programa de fisioterapia em uma paciente no pós-operatório de câncer de mama, buscando uma melhora do quadro álgico, da amplitude de movimento e da força muscular desta paciente. 


\section{Apresentação do caso}

$\infty \times \infty \times \infty \times \infty \times \infty \times \infty \times \infty \times \infty \times \infty \times \infty \times \infty \times \infty \infty$

0 estudo foi delineado pelo método qualiquantitaO estudo foi delineado pelo método qualiquantitativo em saúde, do tipo estudo de caso. A amostra constou de uma paciente atendida no Estágio de Fisioterapia Oncológica da Clínica Escola de Fisioterapia da URI - campus de Erechim, do sexo feminino, 60 anos de idade, estando no segundo mês do pós-operatório de câncer de mama direita. A presente pesquisa cumpriu com os princípios éticos contidos na Declaração de Helsinque, e foi realizada somente após a assinatura do Termo de Consentimento Livre e Esclarecido (TCLE) pela paciente.

Ao primeiro encontro a paciente relatou que durante seus exames de rotina foi detectado um nódulo em sua mama direita e seis meses após realizou mastectomia radical com esvaziamento axilar devido a um carcinoma ductal infiltrante. A paciente relatou muita dor na região axilar e de membro superior direito e região cervical.

Durante a avaliação foi possivel observar que a paciente não apresentava evidências de linfedema em membro superior direito ou no local da cirurgia; cicatriz cirúrgica medindo 25 centímetros, hiperpigmentada, com sensibilidade normal e sem nenhuma alteração.

A partir do quadro apresentado pela paciente, avaliou-se a intensidade da dor através da Escala Visual Analógica da dor (EVA) (esta era verificada no início e término de cada atendimento); perimetria de membros superiores; amplitude de movimento articular ativa de membros superiores com o uso do goniômetro; força muscular de membros superiores por meio da escala de Oxford e do dinamômetro de preensão manual e dor e amplitude de movimento através da Escala de Fulg-Meyer.

Foram realizados 12 atendimentos fisioterapêuticos em oncologia na Clínica Escola de Fisioterapia da URI - campus de Erechim, com duração, em média, de 50 minutos cada, uma vez na semana.

0 tratamento proposto baseou-se nas limitações funcionais avaliadas, isto é, algia em membro superior direito, região axilar direita e cervical; tensão muscular em membros superiores e em cintura escapular; diminuição da amplitude de movimento em membros superiores e diminuição da força muscular em membro superior direito.

As condutas analgésicas utilizadas foram aplicação de TENS, interferencial, realização de massoterapia e pompage principalmente em região de membro superior direito e cervical. Para aumento da amplitude de movimento, foi realizado mobilização articular de Maitland nas articulações glenoumeral e escapulotorácica, alongamentos passivos e ativos para todos os grupos musculares de membros superiores e de coluna cervical, exercícios de ADM passivos, ativo-assistidos e ativos para membros superiores, com uso de bastão, toalha, roldana, bolinha, bola suiça além de exercícios pendulares. Também foi realizada drenagem linfática na região axilar e de membro superior direito. Além disso, foram passadas orientações à paciente em relação aos cuidados que esta deveria ter em relação ao membro superior e sobre a autodrenagem, assim como exercícios para que a ela pudesse realizar em seu domicílio.

Em relação à dor, avaliada pela Escala Visual Analógica, referida em região axilar e de membro superior direito e cervical, foi possível observar que esta diminuía de um a dois pontos do início para o término da sessão. Entretanto, a dor da paciente oscilava de uma sessão para a outra, mesmo assim se observou uma redução progressiva do quadro álgico, conforme mostrado na figura 1 .

A perimetria foi realizada em ambos os antebraços, partindo do processo estiloide do rádio, e em ambos os braços, partindo do epicôndilo lateral do úmero. Foi possível observar que não havia aumento da circunferência do membro ipsilateral a cirurgia, sendo que em algumas medições este se apresentava menor que o membro contralateral, evidenciando que não havia presença de linfedema. Após o término do tratamento, observou-se uma diminuição da circunferência tanto de antebraço como do braço de ambos os membros superiores, evidenciando uma perda de massa muscular, conforme descrito na tabela 1 .

Já em relação à amplitude de movimento ativa da paciente, observou-se na avaliação inicial uma grande limitação em ambos os membros superiores, principalmente nos movimentos do ombro (Tabela 1 ). Após as 12 sessões realizadas, obser- 
vou-se um aumento e/ou manutenção da amplitude de movimento conforme descrição na tabela 2 .

No que se refere à força muscular, a paciente apresentava grau 5 em toda a musculatura de membro superior esquerdo e grau 4 em toda musculatura de membro superior direito, segundo escala de Oxford. Após o tratamento realizado, foi possivel observar a manutenção do mesmo nível de força em ambos os membros superiores. 0 mesmo ocorreu na avaliação da força através da dinamometria, os valores obtidos inicialmente (direita: $0,5 \mathrm{kgf} / \mathrm{cm}^{2}$; esquerda: $0,9 \mathrm{kgf} / \mathrm{cm}^{2}$ ) mantiveram-se na avaliação final.

Já em relação a escala de Fugl-Meyer, que correlaciona a dor com o grau de amplitude de movimento, podemos observar que o membro superior direito apresentava uma limitação principalmente nos movimentos de ombro no momento da avaliação, sendo que após as 12 sessões houve uma melhora tanto em relação à dor, quanto em relação à amplitude de movimento avaliada, conforme tabela 3.

\section{Discussão}

$\infty \times \infty \times \infty \times \infty \times \infty \times \infty \times \infty \times \infty \times \infty \times \infty \times \infty \times \infty \infty$

0 presente estudo objetivou verificar os benefícios proporcionados por um programa de fisioterapia no pós-operatório de câncer de mama. A paciente abordada estava no segundo mês do pós-operatório, ou seja, estava na fase inicial do tratamento. Para Faria ${ }^{4}$, a fisioterapia oncológica precoce desempenha um papel importante na prevenção e minimização dos efeitos adversos do tratamento do câncer de mama, reduzindo os riscos de complicações e restaurando a integridade cinético-funcional dos órgãos e sistemas.

Segundo Nascimento, Oliveira, Oliveira e Amaral $^{6}$, a fisioterapia é fundamental no pós-operatório, objetivando a reabilitação, prevenção e recuperação dos movimentos do membro superior. Além disso, a fisioterapia iniciada nos primeiros dias após a cirurgia traz inúmeras vantagens, como prevenção de linfedema, retrações e disfunção do ombro, pelo aumento de volume de sangue e linfa drenados, e do próprio encorajamento da paciente em reassumir as atividades normais. ${ }^{7-8}$

Ao avaliarmos a paciente verificamos que ela apresentava dor na região axilar e de membro su- perior direito e cervical, diminuição da amplitude de movimento em ambos os membros superiores e fraqueza muscular no membro superior ipsilateral à cirurgia. A dor, as disfunções musculoesqueléticas no ombro homolateral à cirurgia, a ansiedade e o medo são frequentemente encontrados nas mulheres que estão no pós-operatório de câncer de mama. ${ }^{6}$

Segundo Faria ${ }^{4}$, a dor é uma das principais e mais frequentes queixas da paciente, devendo ser valorizada, controlada e tratada em todas as etapas da doença, já que esta compromete negativamente o cotidiano e a qualidade de vida destas pacientes. ${ }^{9}$ Além disso, a sensação de dor na ferida operatória e na região cervical está presente principalmente no pós-operatório imediato. ${ }^{10}$ Durante as sessões foi possivel observar que a dor da paciente diminuía ao compararmos o início com o final da sessão, e ao término das 12 sessões foi possível observar uma diminuição do quadro álgico da paciente.

Por meio da perimetria de membros superiores observamos que a paciente não apresentava linfedema quando comparado ao membro contralateral. Além disso, em algumas medições o membro homolateral apresentava-se com diâmetro menor que o membro contralateral, evidenciando uma perda de massa muscular, confirmada na reavaliação, que mostrou uma diminuição do diâmetro de ambos os membros superiores.

O linfedema é um problema que gera isolamento social e é estigmatizante, e seus sintomas no braço estão relacionados aos sintomas do linfedema, como restrição de mobilidade, dor e edema, limitando as atividades funcionais. ${ }^{11}$ Estudos demonstraram que a prevalência de linfedema aumenta com o tempo decorrido desde a radioterapia, variando $0-23 \%$ nos dois primeiros anos para $45 \%$ após 15 anos ou mais. ${ }^{12}$ Nascimento, Oliveira, Oliveira e Amaral ${ }^{6}$ observaram em seu estudo um aumento da frequência do linfedema no segundo ano de pós-operatório. Devido ao fato da paciente estar no momento da reavaliação no quinto mês de pós-operatório, talvez ainda não seja possível detectar a presença de um linfedema, já que este surge em fases mais tardias, por isso a importância de medidas preventivas e orientações a fim de evitá-lo. 
A diminuição da ADM é muito relatada na literatura no pós-operatório de câncer de mama. De acordo com Camargo e Marx ${ }^{3}$, é comum a articulação do ombro ser a mais afetada devido à hipomobilidade do membro superior homolateral à cirurgia. Os movimentos de abdução e flexão do ombro geralmente são os mais afetados; isso ocorre geralmente devido à dor e também devido à cicatriz cirúrgica, já que a maioria das mulheres submetidas ao tratamento cirúrgico não movimentam o membro superior por medo de deiscência da cirurgia. ${ }^{2}$

Em nosso estudo foi possível observar que a limitação da amplitude de movimento ocorreu tanto no membro superior do lado afetado quanto do lado não afetado. Bregagnol e Dias ${ }^{2}$ também verificaram alteração em relação a ADM de flexão e abdução do ombro do lado não afetado, quando comparado o momento pré com o pós-operatório imediato. Segundo estes autores, isso ocorre pela dor e também pela cicatriz cirúrgica, que leva a uma limitação da movimentação do lado contralateral à cirurgia.

Após as 12 sessões foi possivel observar um aumento da amplitude de movimento em ambos os membros superiores, avaliada através da goniometria e também por intermédio da escala de Full-Meyer. Rett, Mesquita, Mendonça, Moura e DeSantana ${ }^{9}$ realizaram um programa de fisioterapia com 39 mulheres submetidas ao tratamento cirúrgico do câncer de mama, o programa envolveu alongamentos, exercícios ativos livres e exercícios ativos assistidos de membros superiores. Após 20 sessões eles verificaram que a cinesioterapia meIhorou a ADM e reduziu a dor no MS, especialmente no início da intervenção $(p=0,033)$, evidenciando a importância da abordagem inicial da fisioterapia.

Pinto e Silva, Derchain, Rezende, Cabello e Martine $z^{7}$ verificaram que a realização de exercícios ativos e de alongamento, com amplitude livre desde o primeiro dia de pós-operatório em mulheres com câncer de mama, permite boa recuperação da capacidade funcional do ombro sem aumento de seroma ou deiscência.

Segundo Nascimento, Oliveira, Oliveira e Amaral $^{6}$, a ADM do ombro melhora mais evidentemente entre o terceiro mês e o primeiro ano após a cirurgia, possivelmente pela eliminação dos efeitos agudos do tratamento cirúrgico e/ou radioterápico. Para Rett, Santos, Mendonça, Oliveira, DeSanta$n a^{13}$, a cinesioterapia é importante para o aumento da ADM, além de reduzir a dor no início do tratamento e mantê-la controlada por longo tempo.

Em relação à força muscular observamos que esta se manteve a mesma após as 12 sessões. Entretanto, se correlacionarmos o fato de que houve uma diminuição do diâmetro de membros superiores avaliado pela perimetria, o que nos leva a pensar em uma perda de massa muscular, o fato de não ter ocorrido perda de força muscular é um bom resultado. Além disso, não realizamos nenhum exercício de fortalecimento muscular, já que os principais problemas encontrados eram a dor e a limitação da ADM.

Vários estudos mostraram que a fisioterapia no pós-operatório é benéfica para o ganho de força muscular, mas os estudos que realizaram treinamento de força iniciaram mais tardiamente. Como o estudo de Leite, Knorst, Lima, Zerwes e Frison ${ }^{10}$, que verificaram um aumento de força muscular após um protocolo de 8 semanas com 10 mulheres submetidas a tratamento cirúrgico e adjuvante para câncer de mama.

Para Lahoz, Nyssen, Correia, Garcia e Driusso ${ }^{14}$, a associação entre a diminuição da amplitude de movimento, diminuição da força muscular com a queixa de dor no ombro promove um impacto negativo sobre a qualidade de vida destas pacientes. A qualidade de vida não foi avaliada em nosso estudo, entretanto foi possível observar os sentimentos de tristeza, angústia, medo que a paciente relatava, afetando muito a qualidade de vida desta. Isso confirma mais uma vez a importância da fisioterapia, já que a melhora dos sintomas físicos melhora consequentemente o estado emocional destes pacientes ${ }^{15}$.

\section{Considerações finais}

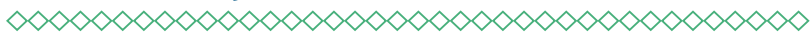

Verificou-se que o protocolo de tratamento fisioterapêutico do presente estudo foi eficaz no pós-operatório de câncer de mama, proporcionando diminuição da dor, prevenção do linfedema, aumento da amplitude de movimento de membros superiores, sem alteração na força muscular. 


\section{Referências}

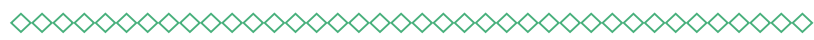

1. INCA - Instituto Nacional do Câncer. Rio de Janeiro: Ministério da Saúde; 2013 [acesso em 28 jun 2013] Disponível em: http://www2.inca.gov. $\mathrm{br} / \mathrm{wps} / \mathrm{wcm} / \mathrm{connect} /$ tiposdecancer/site/home / mama/cancer_mama+.

2. Bregagnol RK, Dias AS. Alterações Funcionais em Mulheres Submetidas à Cirurgia de Mama com Linfadenectomia Axilar Total. Rev Bras de Cancerologia. 2010;56(1):25-33.

3. Camargo MC, Marx, AG. Reabilitação física no câncer de mama. São Paulo: Roca; 2000.

4. Faria L. As práticas do cuidar na oncologia: a experiência da fisioterapia em pacientes com câncer de mama. História, Ciências, Saúde. 2010;17(1):69-87.

5. Jammal MP, Machado ARM, Rodrigues LR. Fisioterapia na reabilitação de mulheres operadas por câncer de mama. 0 Mundo da Saúde São Paulo. 2008;32(4):506-10.

6. Nascimento SL, Oliveira RR, Oliveira MMF, Amaral MTP. Complicações e condutas fisioterapêuticas após cirurgia por câncer de mama: estudo retrospectivo. Fisioter Pesq. 2012;19(3):248-55.

7. Pinto e Silva MP, Derchain SFM, Rezende L, Cabello C, Martinez EZ. Movimento do ombro após cirurgia por carcinoma invasor da mama: estudo randomizado prospectivo controlado de exercícios livres versus limitados a $90^{\circ}$ no pós-operatório. RBG0. 2004;26(2):125-30.

8. Rezende LF, Franco RL, Gurgel MSC. Fisioterapia aplicada à fase pós-operatório do câncer de mama: o que considerar. Rev. Ciên. Méd. 2005; 14(3):295-302.
9. Rett MT, Mesquita PJ, Mendonça ARC, Moura DP, DeSantana JM. A cinesioterapia reduz a dor no membro superior de mulheres submetidas à mastectomia ou quadrantectomia. Rev Dor. 2012;13(3):201-7.

10. Leite GT, Knorst MR, Lima CHL, Zerwes FP, Frison VB. Fisioterapia em oncologia mamária: qualidade de vida e evolução clínico funcional. Rev. Ciên. \& Saúde. 2010;3(1):14-21.

11. Alegrance FC, Souza CB, Mazzei RL. Qualidade de Vida e Estratégias de Enfrentamento em Mulheres com e sem Linfedema PósCâncer de Mama. Rev. Bras. de Cancerologia. 2010;56(3):341-51.

12. Oliveira MMF, Gurgel MSC, Miranda MS, Okubo MA, Feijó LFA, Souza GA. Efficacy of shoulder exercises on locoregional complications in women undergoing radiotherapy for breast cancer: clinical trial. Rev. Bras. de Fisioterapia. 2009;13(2):136-43.

13. Rett MT, Santos AKG, Mendonça ACR, Oliveira IA, DeSantana JM. Efeito da fisioterapia no desempenho funcional do membro superior no pós-operatório de câncer de mama. Rev. Ciên. \& Saú. 2013;6(1):18-24.

14. Lahoz MA, Nyssen SM, Correia GN, Garcia APU, Driusso P. Capacidade Funcional e Qualidade de Vida em Mulheres Pós-Mastectomizadas. Rev. Bras. de Cancerologia. 2010;56(4):423-30.

15. Alves NC. Câncer de mama e corpo feminino: análise a partir de depoimentos orais. Rev. de Psic. da UNESP. 2011;10(2):28-35. 


\section{Anexos}

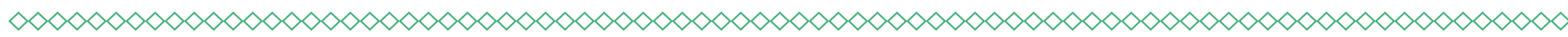

Figura 1 - Avaliação da dor em membro superior direito e cervical pré e pós-intervenção fisioterapêutica

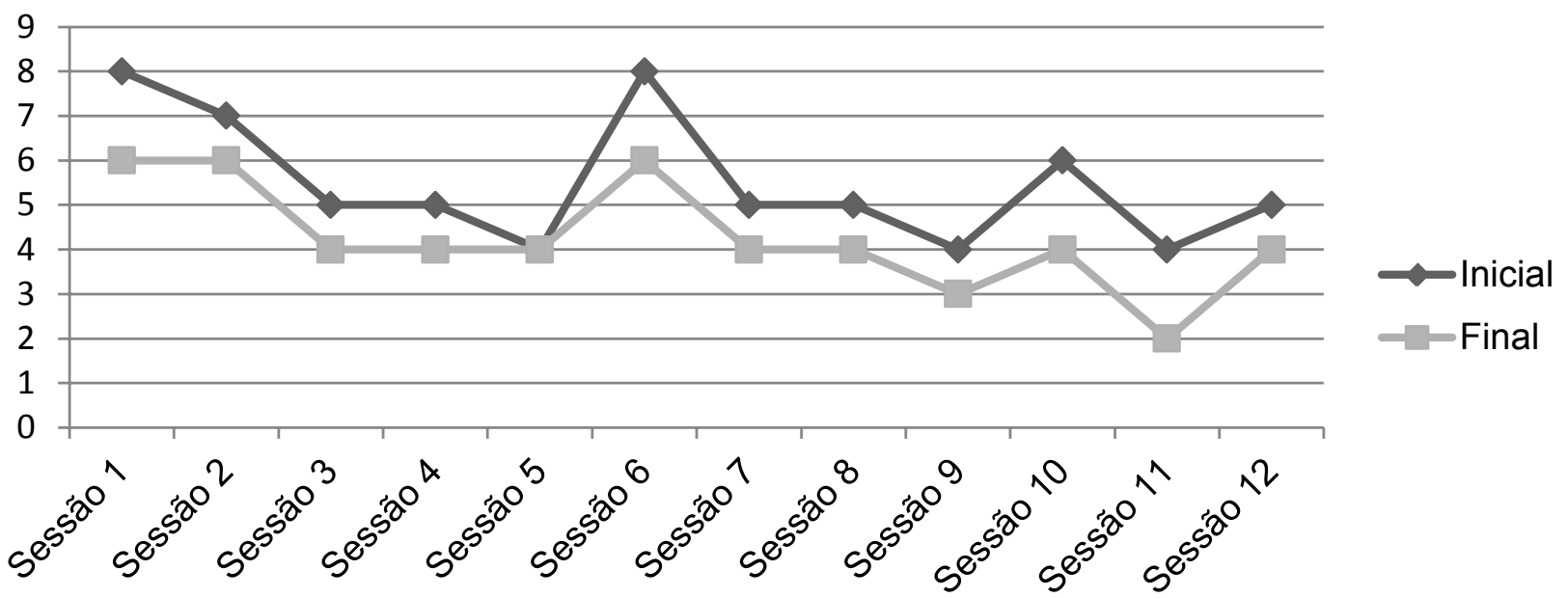

elaboração dos autores.

(clique para voltar ao texto)

Tabela 1 - Perimetria de membros superiores pré e pós-intervenção

\begin{tabular}{ccccccccc} 
& \multicolumn{3}{c}{ PRÉ } & \multicolumn{2}{c}{ PÓS } & \multicolumn{2}{c}{ PRÉ } & \multicolumn{2}{c}{ PÓS } \\
\cline { 2 - 8 } & Anteb. & Braço & Anteb. & Braço & Anteb. & Braço & Anteb. & Braço \\
\hline $0 \mathrm{~cm}$ & 18,5 & 27 & 17,5 & 26 & 17,5 & 27,5 & 17,5 & 26,5 \\
$5 \mathrm{~cm}$ & 20 & 29 & 18 & 27,5 & 19,5 & 29 & 18,5 & 27,5 \\
$10 \mathrm{~cm}$ & 23 & 30,5 & 22 & 29,5 & 24 & 30 & 22,5 & 29,5 \\
$15 \mathrm{~cm}$ & 25,5 & 32 & 24,5 & 30 & 25,5 & 31,5 & 25 & 30 \\
$20 \mathrm{~cm}$ & 26 & & 25 & & 27 & & 26,5 & \\
\hline
\end{tabular}

Obs.: antebraço: início no processo estiloide; braço: epicôndilo lateral.

Fonte: elaboração dos autores.

(clique para voltar ao texto) 
Tabela 2 - Avaliação da amplitude de movimento articular de membros superiores bilateralmente pré e pós-intervenção

\begin{tabular}{lcccc} 
& \multicolumn{2}{c}{ DIREITO } & & \multicolumn{2}{c}{ PSQ́́ } & PÓS \\
\hline OMBRO & & PÓS & 130 & 180 \\
Flexão & 100 & 120 & 60 & 60 \\
Extensão & 60 & 60 & 130 & 160 \\
Abdução & 100 & 125 & 70 & 90 \\
Rot. Interna & 40 & 55 & 70 & 90 \\
Rot. Externa & 50 & 50 & 140 & 140 \\
\hline COTOVELO & & & 0 & 0 \\
Flexão & 140 & 140 & & 90 \\
Extensão & 0 & 0 & 90 & 90 \\
\hline ANTEBRAÇO & 90 & 90 & 90 & \\
Supinação & 90 & 90 & & \\
Pronação & & & & \\
\hline
\end{tabular}

Fonte: elaboração dos autores. 
Tabela 3 - Escala de Fulg-Meyer pré e pós-tratamento

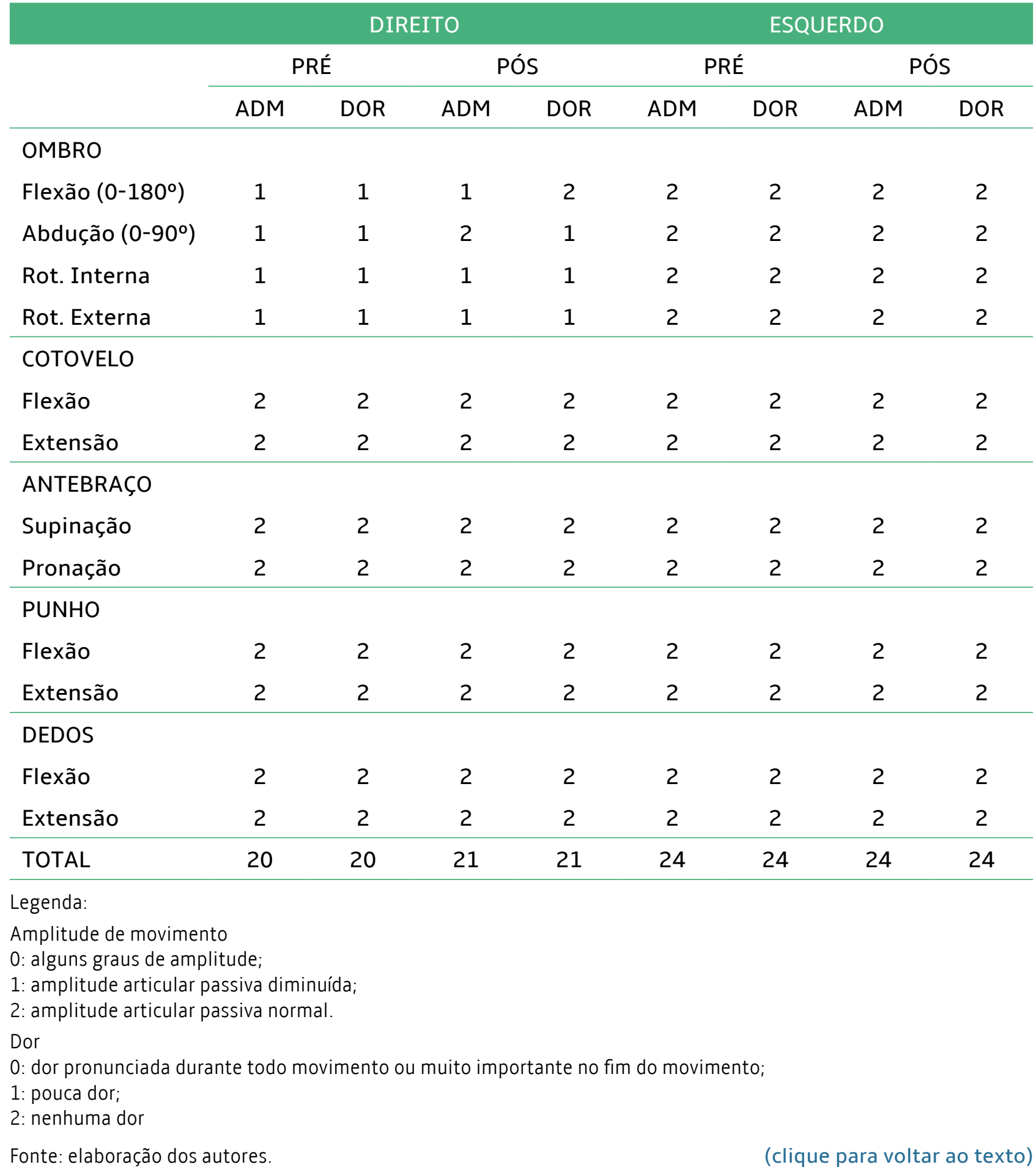

